\title{
Chebyshev Approximations for the Riemann Zeta Function
}

\author{
By W. J. Cody,* K. E. Hillstrom,* and Henry C. Thacher, Jr.**
}

\begin{abstract}
This paper presents well-conditioned rational Chebyshev approximations, involving at most one exponentiation, for computation of either $\zeta(s)$ or $\zeta(s)-1, .5 \leqq$ $s \leqq 55$, for up to 20 significant figures. The logarithmic error is required in one case. An algorithm for the Hurwitz zeta function, and an example of nearly double degeneracy are also given.
\end{abstract}

1. Introduction. The Riemann zeta function is defined by

$$
\zeta(s)=\sum_{k=1}^{\infty} k^{-s} \quad(\operatorname{Re}(s)>1)
$$

or by the power series expansion

$$
\zeta(s)=\frac{1}{s-1}+\sum_{n=0}^{\infty} \frac{(-1)^{n}}{n !} \gamma_{n}(s-1)^{n} \quad(\operatorname{Re}(s)>0)
$$

where

$$
\gamma_{n}=\lim _{m \rightarrow \infty}\left\{\sum_{k=1}^{m} \frac{(\ln k)^{n}}{k}-\frac{(\ln m)^{n+1}}{n+1}\right\}
$$

It is an analytic function of $s$, regular throughout the complex plane except for a simple pole of residue 1 at $s=1$. The zeta function satisfies the functional equation

$$
\zeta(1-s)=2^{1-s} \pi^{-s} \cos \left(\frac{1}{2} \pi s\right) \Gamma(s) \zeta(s) .
$$

Evaluation of the function for real $s$ usually involves taking a partial sum of (1.1) and applying the Euler-Maclaurin summation formula to the remainder. While this procedure is theoretically valid for all $s>-2 n-1$, where $n$ terms of the EulerMaclaurin summation formula are used, there is serious cancellation error for $s<1.5$. However, the reflection formula, Eq. (1.3), can be used for $s<.5$, while Thacher [7] has recently used Eq. (1.2) as a basis for expansions in Chebyshev polynomials valid both for $\frac{1}{2} \leqq s \leqq \frac{3}{2}$ and for $1 \leqq s \leqq 2$. For $s \geqq 2$, it is still necessary to evaluate a partial sum of the series (1.1). The process involves an exponentiation for each new term added to the sum, and is therefore quite slow. This paper presents rational Chebyshev approximations for evaluating $\zeta(s)$ or $\zeta(s)-1$ for up to 20S without any exponentiation for $.5 \leqq s \leqq 11$, and with only one exponentiation for $11 \leqq s \leqq 55$.

Received August 7, 1970, revised December 21, 1970.

AMS 1969 subject classifications. Primary 3304, 3345, 6525; Secondary 4117, 4140, 6520.

Key words and phrases. Rational Chebyshev approximations, Riemann zeta function, Hurwitz zeta function, logarithmic error, near degeneracy.

* Work performed under the auspices of the U. S. Atomic Energy Commission.

** Work supported in part by the U. S. Atomic Energy Commission and in part by the University of Notre Dame. 
The approximation forms and the values of $s$ for which they are used are

$$
\begin{aligned}
\zeta(s) & \simeq R_{l m}(s) /(s-1), & .5 \leqq s \leqq 5, \\
\zeta(s)-1 & \simeq R_{l m}(s), & 5 \leqq s \leqq 11, \\
& \simeq 2^{-s+(1 / s) R_{l m}(1 / s)}, & 11 \leqq s \leqq 25,25 \leqq s \leqq 55,
\end{aligned}
$$

where the $R_{l m}(s)$ are rational functions of degree $l$ in the numerator and $m$ in the denominator. The maximum error was computed relative to $\zeta(s)$ for the first interval, and relative to $\zeta(s)-1$ for the others.

2. Computational of Reference Values. Reference function values for the generation of the approximations were calculated for $.5 \leqq s \leqq 1.5$ from the coefficients given by Thacher [7], and for the other $s$ from a modification of the above-described technique based on the Euler-Maclaurin summation formula. The modification involves a method for estimating the number of terms needed in the partial sum of (1.1).

The Euler-Maclaurin formula applied to the Dirichlet series for the Hurwitz zeta function,

$$
\zeta(s ; \alpha)=\sum_{k=0}^{\infty}(k+\alpha)^{-s}, \quad \alpha>0, \operatorname{Re}(s)>1,
$$

gives

$$
(s-1) \zeta(s ; \alpha)=\frac{2 \alpha+s-1}{2 \alpha^{s}}+\sum_{k=1}^{[n / 2]} \frac{B_{2 k}}{(2 k) !} \frac{\Gamma(s+2 k-1)}{\Gamma(s-1) \alpha^{s+2 k-1}}+R_{n},
$$

where

$$
R_{n}=\frac{(-1)^{n+1} \Gamma(s+n)}{n ! \Gamma(s-1)} \int_{0}^{\infty} \frac{\hat{B}_{n}(-t) d t}{(\alpha+t)^{s+n}}
$$

and $\hat{B}_{n}(x)$ is the periodic extension of the $n$th Bernoulli polynomial. Since $(\alpha+t)^{s+n}>0$ for $t>-\alpha$, the mean value theorem can be used to obtain

$$
R_{n}=(-1)^{n+1} \frac{\Gamma(s+n-1)}{n ! \Gamma(s-1)} \frac{B_{n}(\xi)}{\alpha^{s+n-1}}, \quad 0 \leqq \xi \leqq 1 .
$$

Thus

$$
\left|R_{n}\right| \leqq \frac{1}{n !} \prod_{k=1}^{n} \frac{(s+k-2) M_{n}}{|\alpha|^{s+n-1}}
$$

where

$$
M_{n}=\max _{0 \leqq x \leqq 1}\left|B_{n}(x)\right| .
$$

Letting $G$ and $A$ denote the geometric and arithmetic means of the quantities $\{(s+k-2)\}$, and using the arithmetic-geometric mean inequality, we have

$$
\prod_{k=1}^{n}(s+k-2)=G^{n} \leqq A^{n}=\left\{\sum_{k=1}^{n} \frac{(s+k-2)}{n}\right\}^{n}=\left(s+\frac{n-3}{2}\right)^{n},
$$


so that

$$
\left|R_{n}\right| \leqq \frac{1}{n !}\left(s+\frac{n-3}{2}\right)^{n} \frac{M_{n}}{|\alpha|^{s+n-1}} .
$$

Lehmer [4] discusses the extrema of the Bernoulli polynomials and shows that $M_{n}=\left|B_{n}\right|$ for $n$ even. For $n$ odd, he gives $11 D$ values of $M_{n}$, for $n \leqq 13$, as well as relatively sharp asymptotic formulas for larger $n$. We thus see that [ $n / 2]$ terms of (2.2) will approximate $(s-1) \zeta(s ; \alpha)$ with an absolute error less than $10^{-D}$ provided that

$$
|\alpha| \geqq\left\{\left(s+\frac{n-3}{2}\right)^{n} \frac{M_{n}}{n !} 10^{D}\right\}^{1 /(s+n-1)} .
$$

In principle, then, one can obtain $(s-1) \zeta(s ; a)$ to any desired accuracy by selecting $n$ and $m$ so that (2.8) is satisfied for $\alpha=a+m$, evaluating $(s-1) \zeta(s ; \alpha)$ by (2.2), and finally computing $(s-1) \zeta(s ; a)$ by adding $(s-1)$ times the appropriate partial sum of the Dirichlet series for $\zeta(s ; a)$. The procedure is valid for $\operatorname{Re}(s)>0$.

The most efficient value of $n$ depends upon $s$ and $D$. In our calculations, we did not vary this parameter, but gave it the constant value 13 , for which

$$
m=\left[\left\{8.40798 \times 10^{D-11}(s+5)^{13}\right\}^{1 /(s+12)}+1-a\right],
$$

where [ ] denotes "the integer part of." The variable order of the partial sum prevented the use of Markman's economical method [5] of reducing the necessary number of exponentiations in (2.2).

All computations were carried out on a CDC 3600 in $25 \mathrm{~S}$ arithmetic. Extensive checking against tables and by overlapping of methods shows that our master routines were accurate to roughly a minimum of $23 \mathrm{~S}$.

3. Generation of the Approximations. The various approximations were generated in 25S floating-point arithmetic on a CDC 3600 using standard versions of the Remes algorithm [2]. With two exceptions, the computations were straightforward.

The first exception was the analysis relating to the approximation form for the last two intervals. The quantity

$$
\delta(s)=\frac{\zeta(s)-1-2^{-s+(1 / s) R_{l m}(1 / s)}}{\zeta(s)-1}
$$

is the error of approximation relative to $\zeta(s)-1$. However, the basic Remes algorithm is limited to error expressions of the form

$$
\Delta(s)=\frac{f(s)-R_{l m}(\phi(x))}{g(x)} .
$$

Hence, we must modify (3.1). By letting

$$
\delta(s)=1-2^{-d(s)},
$$

we find that $d(s)$ has the form (3.2) with

$$
f(s)=s[\ln (\zeta(s)-1) / \ln 2+s], \quad g(s)=s,
$$

and

$$
\phi(s)=1 / s .
$$


We note that the local extrema of $d(s)$ and $\delta(s)$ occur for the same values of $s$, and that

$$
\hat{\delta}(s)=(-\ln 2) d(s)=\ln (1-\delta(s)) \simeq \delta-\frac{1}{2} \delta^{2},
$$

where $\hat{\delta}(s)$ is the "logarithmic error" discussed by King and Phillips [3] and Sterbenz and Fike [6]. The logarithmic error has been associated primarily with obtaining starting values for various Newton iteration schemes. However, we can use $\hat{\delta}(s)$ in the Remes algorithm since it approximates the Chebyshev error $\delta(s)$ to within terms of order $\delta^{2}(s)$, an error that is swamped by normal roundoff in the Remes algorithm itself whenever $\delta(s)$ is small.

The second anomaly occurred in the computation of $R_{88}(1 / s)$ for the interval $[11,25]$. Although the error curves for $R_{68}$ and $R_{77}$ appear to be standard, $R_{88}$ is nearly doubly degenerate. The method of artificial poles [2] determined the Chebyshev error for $R_{88}$ as approximately $5.2 \times 10^{-17}$, with the error curve still not leveled. At this point, the denominator had among its zeros the values

$$
s_{1}=.0371111862 \text { and } s_{2}=.13063202 \text {. }
$$

Corresponding zeros in the numerator were $s_{1}+\left(1 \times 10^{-10}\right)$ and $s_{2}+\left(2 \times 10^{-8}\right)$. To our knowledge, this is the first case of nearly double degeneracy that has occurred in practice.

4. Results. Table I lists the values of

$$
E_{l m}=-100 \log _{10} \delta_{l m}
$$

for selected segments of the $L_{\infty}$ Walsh arrays. The minimax error $\delta_{l m}$ of approximation by $R_{l m}$ is the error relative to $\zeta(s)$ for the interval [.5, 5], and relative to $\zeta(s)-1$ for the other intervals.

Tables II-V present the approximations giving accuracies most appropriate for computers in use today. The coefficients are given to accuracy slightly greater than that justified by the approximation errors, but reasonable additional rounding should not greatly affect the overall accuracies. Each approximation listed, using the coefficients just as they appear here, was tested for random arguments against the master function routines, and the stated accuracies were all verified.

There are a few anomalies present in the Walsh array. Nonstandard error curves are flagged in Table I. Usually, a nearly degenerate case is signalled by the presence of a nonstandard error curve for the approximation that is one degree lower in both numerator and denominator. Although as previously mentioned, $R_{77}$ for the interval $[11,25]$ has a standard error curve, $R_{88}$ is nearly doubly degenerate. This troublesome approximation is not given in Table IV. Instead, the nondiagonal element $R_{79}$ is given.

With a little care, computer subroutines returning almost full machine precision values of $\zeta(s)$ and of $\zeta(s)-1$ can be written using these approximations. One troublesome computation is that for $\zeta(s)-1$ for $.5 \leqq s \leqq 5$. If one uses

$$
(s-1) \zeta(s) \simeq R_{l m}(s) \equiv \sum_{i=0}^{l} p_{i} s^{i} / \sum_{i=0}^{m} q_{i} s^{i}, \quad .5 \leqq s \leqq 5,
$$


TABLE I

$$
\begin{gathered}
E_{\ell m}=-100 \log _{10} \delta_{\ell m} \\
.5 \leq s \leq 5 .
\end{gathered}
$$

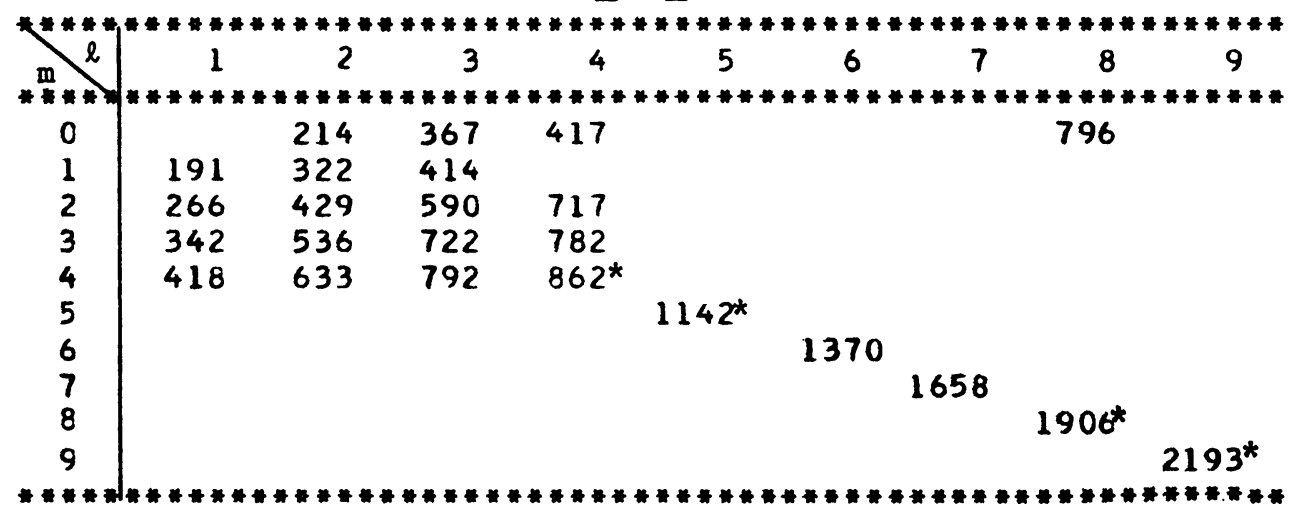

$$
5 \leq s \leq 11
$$

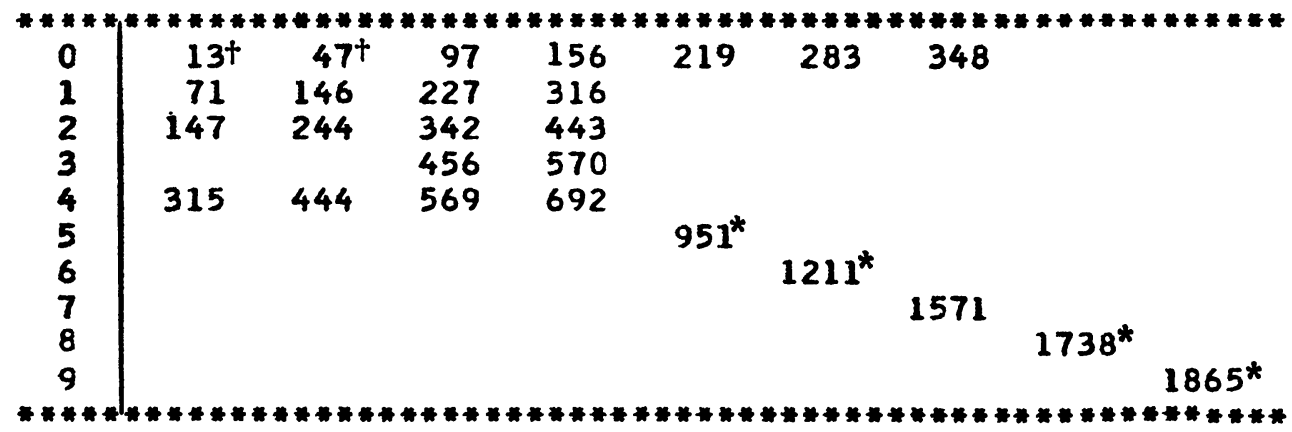

${ }^{t_{\text {Nonstandard }} \text { error curve. }}$

*Coefficients for these approximations only are given in Tables II-V. 
TABLE I (cont'd)

$$
11 \leq \mathrm{s} \leq 25
$$

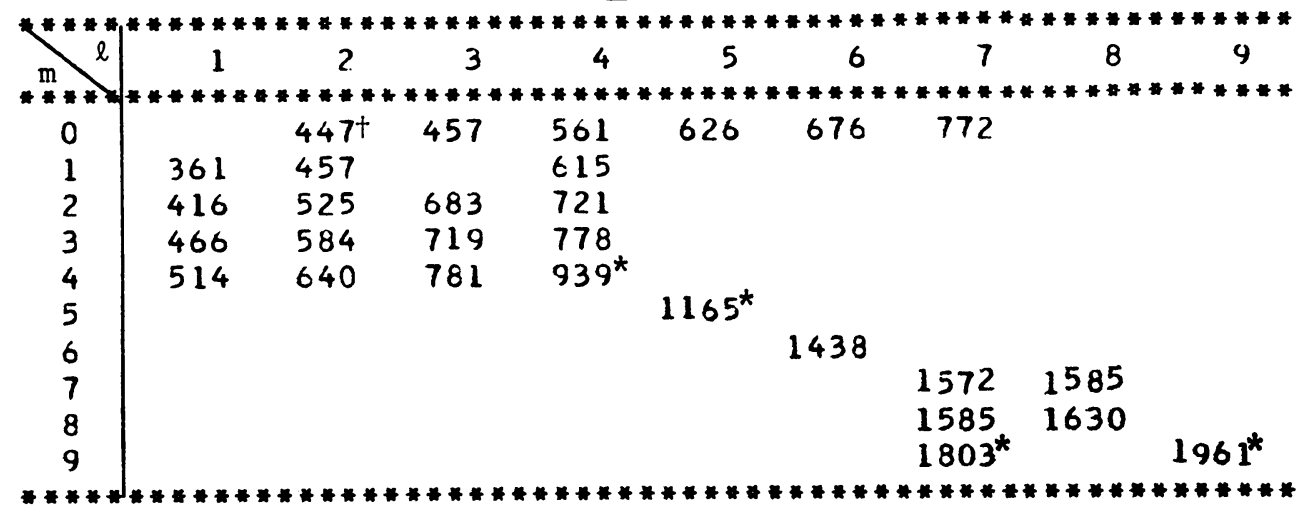

$25 \leq s \leq 55$

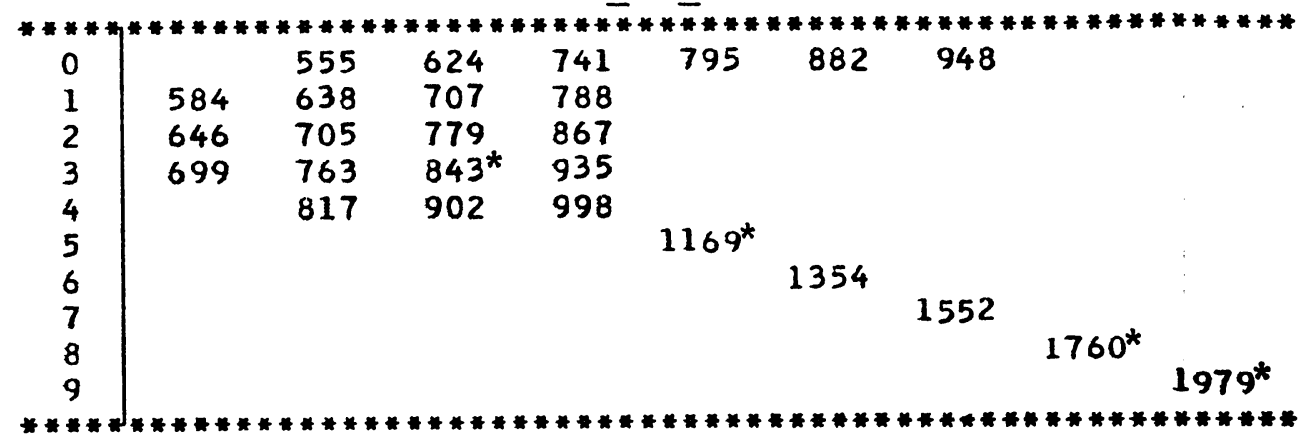

†onstandard error curve.

* Coefficients for these approximations only are given in Tables II-V. 
TABLE II

$$
(s-1) \zeta(s) \simeq \sum_{j=0}^{n} p_{j} s^{j} / \sum_{j=0}^{n} q_{j} s^{j}, \quad .5 \leq s \leq 5
$$

\begin{tabular}{|c|c|c|c|c|c|c|c|c|c|c|c|c|c|}
\hline & j & \multicolumn{6}{|c|}{$\mathrm{p}_{j}$} & \multicolumn{6}{|c|}{$q_{j}$} \\
\hline & $\begin{array}{l}0 \\
1 \\
2 \\
3 \\
4\end{array} \mid$ & $\begin{array}{l}-1.32899 \\
-1.70341 \\
-7.70056 \\
-1.42561 \\
-8.36940\end{array}$ & $\begin{array}{l}37437 \\
74205 \\
02483 \\
64640 \\
23543\end{array}$ & & & & $\begin{array}{ll}1 & 04 \\
1 & 04 \\
1 & 03 \\
1 & 03 \\
1 & 01\end{array}$ & $\begin{array}{r}-2.65799 \\
-1.17972 \\
-1.03940 \\
-1.18419 \\
1.00000\end{array}$ & $\begin{array}{l}37266 \\
24222 \\
13777 \\
54886 \\
00000\end{array}$ & & & & 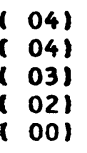 \\
\hline$\underline{\varepsilon}$ & $\begin{array}{l}0 \\
1 \\
2 \\
3 \\
4 \\
5\end{array}$ & $\begin{array}{l}-3.44793 \\
-3.25983 \\
-9.63006 \\
-1.08872 \\
-6.95142 \\
-6.52319\end{array}$ & $\begin{array}{l}47840 \\
44394 \\
98255 \\
52505 \\
48803 \\
89744\end{array}$ & $\begin{array}{l}721 \\
057 \\
869 \\
125 \\
854 \\
728\end{array}$ & & & $\begin{array}{ll}1 & 06 \\
1 & 06 \\
1 & 05 \\
1 & 05 \\
1 & 03 \\
1 & 02\end{array}$ & $\begin{array}{l}-6.8 \\
-7.2 \\
-1.2 \\
-6.2 \\
-7.2 \\
1.2\end{array}$ & $\begin{array}{l}96520 \\
18287 \\
20411 \\
54627 \\
35254 \\
00000\end{array}$ & $\begin{array}{l}340 \\
314 \\
978 \\
536 \\
848 \\
000\end{array}$ & & & 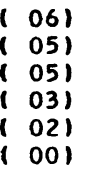 \\
\hline 8 & $\begin{array}{l}0 \\
1 \\
2 \\
3 \\
4 \\
5 \\
6 \\
7 \\
8\end{array}$ & $\begin{array}{l}539 \\
665 \\
147 \\
361 \\
010 \\
957 \\
171 \\
758\end{array}$ & $\begin{array}{l}81214 \\
69320 \\
59183 \\
10024 \\
81243 \\
65854 \\
49902 \\
08431 \\
10040\end{array}$ & $\begin{array}{l}82446 \\
37025 \\
64406 \\
33314 \\
80232 \\
61882 \\
21406 \\
83427 \\
96660\end{array}$ & $\begin{array}{l}39280 \\
11182 \\
10368 \\
86246 \\
98482 \\
51153 \\
08772 \\
12306 \\
65910\end{array}$ & $\begin{array}{ll}9 & 1 \\
5 & 1 \\
3 & 1 \\
9 & 1 \\
4 & 1 \\
5 & 1 \\
8 & 1 \\
1 & 1 \\
9 & 1\end{array}$ & $\begin{array}{ll}1 & 10 \\
1 & 10 \\
1 & 09 \\
1 & 08 \\
1 & 07 \\
1 & 06 \\
1 & 05 \\
1 & 03 \\
1 & 01\end{array}$ & $\begin{array}{r}2 . \\
5 . \\
9 . \\
8 . \\
5 . \\
2 . \\
7 . \\
-2 . \\
1 .\end{array}$ & $\begin{array}{l}62429 \\
56486 \\
03732 \\
66342 \\
17595 \\
12028 \\
89093 \\
57813 \\
00000\end{array}$ & $\begin{array}{l}64846 \\
79590 \\
61233 \\
83289 \\
41920 \\
99137 \\
41537 \\
73772 \\
00000\end{array}$ & $\begin{array}{l}24466 \\
16000 \\
43908 \\
88858 \\
06281 \\
52354 \\
56011 \\
62308 \\
00000\end{array}$ & $\begin{array}{ll}7 & 1 \\
3 & 1 \\
9 & 1 \\
7 & 1 \\
4 & 1 \\
3 & 1 \\
5 & 1 \\
6 & 1 \\
0 & 1\end{array}$ & 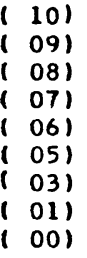 \\
\hline 9 & $\begin{array}{l}0 \\
1 \\
2 \\
3 \\
4 \\
5 \\
6 \\
7\end{array}$ & $\begin{array}{l}9.53904 \\
1.09086 \\
4.57302 \\
9.44640 \\
1.13392 \\
9.69200 \\
6.71953 \\
3.27425 \\
1.09513 \\
2.84456\end{array}$ & $\begin{array}{l}31383 \\
19179 \\
66750 \\
69371 \\
40859 \\
64774 \\
10507 \\
31821 \\
65859 \\
26751\end{array}$ & $\begin{array}{l}75296 \\
76949 \\
30644 \\
30822 \\
96679 \\
06284 \\
96081 \\
83494 \\
34055 \\
69802\end{array}$ & $\begin{array}{l}85073 \\
25970 \\
70069 \\
59763 \\
77378 \\
28141 \\
75927 \\
05394 \\
20435 \\
16448\end{array}$ & $\begin{array}{l}0711 \\
2671 \\
3361 \\
0841 \\
2701 \\
7231 \\
6771 \\
0011 \\
8681 \\
194\end{array}$ & $\begin{array}{ll}1 & 1 \\
1 & 1 \\
1 & 1 \\
1 & 1 \\
1 & 1 \\
1 & 0 \\
1 & 0 \\
1 & 0 \\
1 & 0 \\
1 & 0\end{array}$ & $\begin{array}{l}.90780 \\
.83214 \\
.04516 \\
.22272 \\
.07248 \\
.86833 \\
.42258 \\
13430 \\
.77837 \\
.00000\end{array}$ & $\begin{array}{l}86276 \\
73972 \\
64692 \\
73758 \\
18832 \\
53570 \\
54978 \\
57824 \\
18457 \\
00000\end{array}$ & $\begin{array}{l}75059 \\
70833 \\
48187 \\
61580 \\
75479 \\
00418 \\
70618 \\
29430 \\
74528 \\
00000\end{array}$ & $\begin{array}{l}16848 \\
55022 \\
07276 \\
59174 \\
65542 \\
28875 \\
48187 \\
01348 \\
05958 \\
00000\end{array}$ & $\begin{array}{l}3841 \\
7371 \\
1161 \\
2561 \\
8641 \\
8181 \\
1831 \\
9471 \\
4631 \\
0001\end{array}$ & 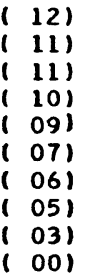 \\
\hline
\end{tabular}


544 W. J. CODY, K. E. HILLSTROM AND HENRY C. THACHER, JR.

TABLE III

$$
\zeta(s) \simeq 1+\sum_{i=0}^{n_{1}} p_{j} T_{j}(t) / \sum_{i=0}^{n_{1}} q_{j} T_{j}(t), \quad t=\frac{s-8}{3}, \quad 5 \leq s \leq 11
$$

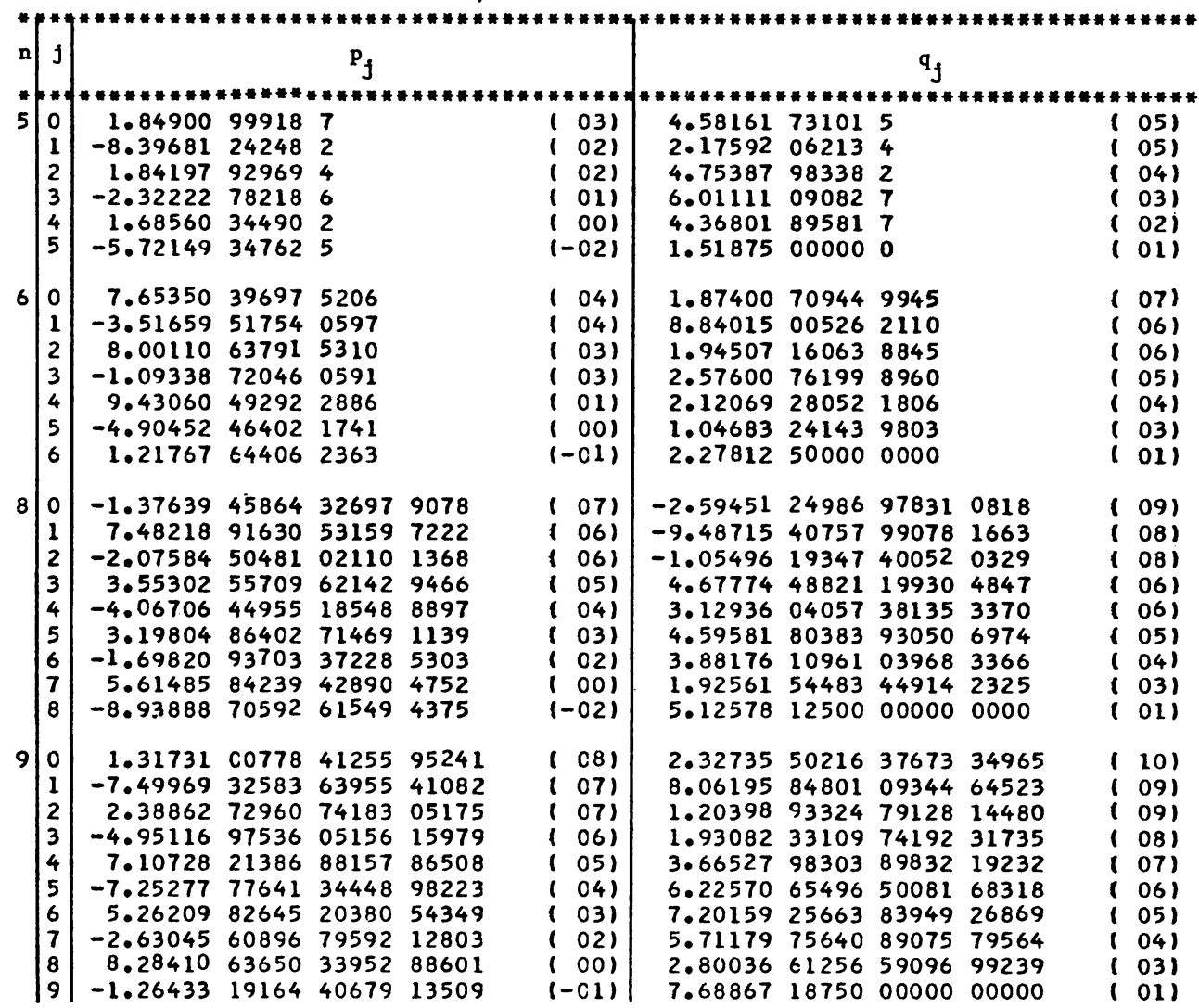


TABLE IV

$$
\zeta(s) \simeq 1+2^{-s+(1 / s)} \cdot \sum_{j=0}^{n} p_{j} s^{-j} / \sum_{j=0}^{n} q_{j} s^{-j}, \quad 11 \leq s \leq 25
$$

\begin{tabular}{|c|c|c|c|c|c|c|c|c|c|}
\hline n $\mathrm{f}$ & & & $\mathrm{p}_{j}$ & & & & $q_{j}$ & & \\
\hline $4\left[\begin{array}{l}0 \\
1 \\
2 \\
3 \\
4\end{array}\right.$ & $\begin{array}{r}2.88915 \\
-3.60673 \\
1.74441 \\
-3.88297 \\
3.36332\end{array}$ & $\begin{array}{l}56312 \\
21549 \\
55297 \\
10518 \\
63994\end{array}$ & $\begin{array}{l}2 \\
6 \\
5 \\
9 \\
4\end{array}$ & & $\begin{array}{l}(-06) \\
(-04) \\
(-02) \\
(-01) \\
(00)\end{array}$ & 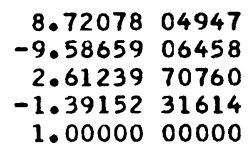 & $\begin{array}{l}2 \\
3 \\
9 \\
9 \\
0\end{array}$ & & $\begin{array}{r}(-05) \\
(-04) \\
(-02) \\
(-02) \\
(00)\end{array}$ \\
\hline $\begin{array}{l}5 \\
0 \\
1 \\
2 \\
3 \\
4 \\
5\end{array}$ & $\begin{array}{r}-6.95395 \\
1.16861 \\
-8.12298 \\
2.92431 \\
-5.46064 \\
4.23648\end{array}$ & $\begin{array}{l}38811 \\
20068 \\
42080 \\
24478 \\
90480 \\
33879\end{array}$ & $\begin{array}{l}340 \\
219 \\
718 \\
121 \\
737 \\
757\end{array}$ & & $\begin{array}{l}(-08) \\
(-05) \\
(-04) \\
(-02) \\
(-01) \\
(00)\end{array}$ & 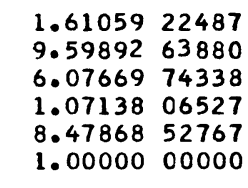 & $\begin{array}{l}913 \\
291 \\
932 \\
427 \\
957 \\
000\end{array}$ & & $\begin{array}{l}(-06) \\
(-06) \\
(-04) \\
(-02) \\
(-02) \\
(00)\end{array}$ \\
\hline $\mid \begin{array}{l}0 \\
1 \\
2 \\
3 \\
4 \\
5 \\
6 \\
7 \\
8 \\
9\end{array}$ & $\begin{array}{r}1.66156 \\
-4.68068 \\
5.83519 \\
-4.17644 \\
1.85468 \\
-5.11288 \\
8.10450 \\
-5.69951\end{array}$ & $\begin{array}{l}48051 \\
82766 \\
72731 \\
01264 \\
42284 \\
80022 \\
23175 \\
94876\end{array}$ & $\begin{array}{l}57746 \\
06545 \\
91470 \\
31456 \\
35979 \\
04902 \\
11003 \\
84789\end{array}$ & $\begin{array}{l}75916 \\
26862 \\
47318 \\
02124 \\
59483 \\
40591 \\
53193 \\
22618\end{array}$ & $\begin{array}{l}(-11) \\
(-09) \\
(-07) \\
(-05) \\
(-03) \\
(-02) \\
(-01) \\
(00)\end{array}$ & 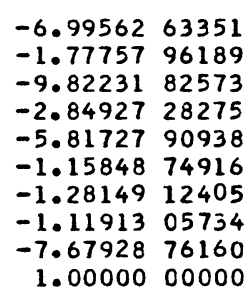 & $\begin{array}{l}91916 \\
51492 \\
40780 \\
90964 \\
80480 \\
97665 \\
19781 \\
90977 \\
46288 \\
00000\end{array}$ & $\begin{array}{l}54964 \\
56941 \\
36442 \\
87594 \\
93531 \\
85807 \\
95742 \\
09324 \\
12537 \\
00000\end{array}$ & $\begin{array}{l}(-10) \\
(-08) \\
(-07) \\
(-05) \\
(-04) \\
(-02) \\
(-01) \\
(00) \\
(-01) \\
(00)\end{array}$ \\
\hline $9 . \mid \begin{array}{l}0 \\
1 \\
2 \\
3 \\
4 \\
5 \\
6 \\
7 \\
8 \\
9\end{array}$ & $\begin{array}{r}6.54074 \\
-1.91182 \\
2.45909 \\
-1.80047 \\
8.06024 \\
-2.17335 \\
3.09201 \\
-1.10135 \\
-1.73589 \\
3.80279\end{array}$ & $\begin{array}{l}87262 \\
33182 \\
27987 \\
53535 \\
99146 \\
07154 \\
82991 \\
65806 \\
22656 \\
09938\end{array}$ & $\begin{array}{l}07601 \\
41692 \\
80779 \\
42409 \\
89416 \\
33717 \\
73503 \\
72497 \\
22043 \\
36744\end{array}$ & 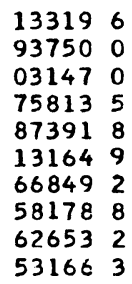 & $\begin{array}{l}(-13) \\
(-10) \\
(-08) \\
(-06) \\
(-05) \\
(-03) \\
(-02) \\
(-01) \\
(00) \\
(00)\end{array}$ & 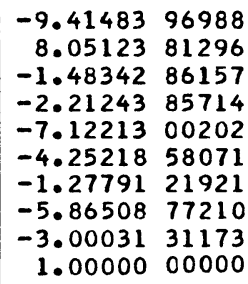 & $\begin{array}{l}23587 \\
05720 \\
21743 \\
34347 \\
26622 \\
71304 \\
98850 \\
99441 \\
95834 \\
00000\end{array}$ & 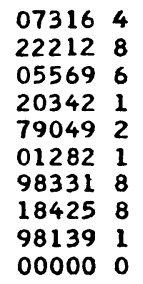 & $\begin{array}{l}(-11) \\
(-10) \\
(-07) \\
(-07) \\
(-05) \\
(-04) \\
(-02) \\
(-02) \\
(-01) \\
(00)\end{array}$ \\
\hline
\end{tabular}


TABLE V

$$
\zeta(s) \simeq 1+2^{-s+(1 / s)} \sum_{j=0}^{n} p_{j} s^{-j} / \sum_{j=0}^{n} q_{j} s^{-j}, \quad 25 \leq s \leq 55
$$

\begin{tabular}{|c|c|c|c|c|c|c|c|c|c|c|c|}
\hline $\mathbf{n}$ & $\mathrm{j}$ & & & $P_{j}$ & & & & & $q_{j}$ & & \\
\hline 3 & {$\left[\begin{array}{l}0 \\
1 \\
2 \\
3\end{array}\right]$} & $\begin{array}{r}4.45020 \\
-6.39481 \\
3.06819 \\
-4.92460\end{array}$ & $\begin{array}{l}40561 \\
80674 \\
07594 \\
44511\end{array}$ & & & $\begin{array}{l}(-09) \\
(-07) \\
(-05) \\
(-04)\end{array}$ & $\begin{array}{r}-9.08360 \\
5.83123 \\
-1.30562 \\
1.00000\end{array}$ & $\begin{array}{l}84927 \\
29118 \\
75204 \\
00000\end{array}$ & & & 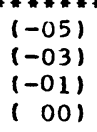 \\
\hline 5 & $\begin{array}{l}0 \\
1 \\
2 \\
3 \\
4 \\
5\end{array}$ & $\begin{array}{r}6.30581 \\
-1.69754 \\
1.84569 \\
-1.01420 \\
2.81957 \\
-3.17620\end{array}$ & $\begin{array}{l}82031 \\
12776 \\
49027 \\
20734 \\
70988 \\
79765\end{array}$ & $\begin{array}{l}926 \\
425 \\
429 \\
917 \\
437 \\
651\end{array}$ & & $\begin{array}{l}(-12) \\
(-09) \\
(-07) \\
(-05) \\
(-04) \\
(-03)\end{array}$ & $\begin{array}{r}-2.45066 \\
2.27263 \\
-9.29701 \\
1.98757 \\
-2.20338 \\
1.00000\end{array}$ & $\begin{array}{l}98983 \\
46889 \\
78224 \\
34180 \\
90078 \\
00000\end{array}$ & $\begin{array}{l}435 \\
318 \\
265 \\
426 \\
903 \\
000\end{array}$ & & $\begin{array}{l}(-07) \\
(-05) \\
(-04) \\
(-02) \\
(-01) \\
(00)\end{array}$ \\
\hline 8 & $\begin{array}{l}0 \\
1 \\
2 \\
3 \\
4 \\
5 \\
6 \\
7 \\
8\end{array}$ & $\begin{array}{r}1.03144 \\
-5.12584 \\
1.12948 \\
-1.44234 \\
1.16824 \\
-6.14975 \\
2.05594 \\
-3.99339 \\
3.45234\end{array}$ & $\begin{array}{l}87718 \\
61396 \\
79419 \\
66537 \\
67698 \\
16799 \\
67798 \\
42939 \\
97673\end{array}$ & $\begin{array}{l}88597 \\
46882 \\
48735 \\
31309 \\
44580 \\
03148 \\
88303 \\
46688 \\
61784\end{array}$ & $\begin{array}{l}1168 \\
4062 \\
4786 \\
5228 \\
9766 \\
0614 \\
2750 \\
6853 \\
5708\end{array}$ & $\begin{array}{l}(-15) \\
(-13) \\
(-10) \\
(-08) \\
(-06) \\
(-05) \\
(-03) \\
(-02) \\
(-01)\end{array}$ & $\begin{array}{r}5.93959 \\
-6.04755 \\
3.64680 \\
-1.29456 \\
3.20189 \\
-5.07801 \\
5.49628 \\
-3.24517 \\
1.00000\end{array}$ & $\begin{array}{l}41728 \\
35907 \\
20866 \\
90556 \\
49847 \\
55709 \\
90788 \\
61115 \\
00000\end{array}$ & $\begin{array}{l}84190 \\
99918 \\
83885 \\
80118 \\
02292 \\
99940 \\
15872 \\
59724 \\
00000\end{array}$ & $\begin{array}{l}5020 \\
0572 \\
6275 \\
1241 \\
5001 \\
7748 \\
6560 \\
1852 \\
0000\end{array}$ & $\begin{array}{l}(-11) \\
(-09) \\
(-07) \\
(-05) \\
(-04) \\
(-03) \\
(-02) \\
(-01) \\
(00)\end{array}$ \\
\hline 9 & $\begin{array}{l}0 \\
1 \\
2 \\
3 \\
4 \\
5 \\
6 \\
7 \\
8 \\
9\end{array}$ & $\begin{array}{r}2.32320 \\
-1.35449 \\
3.55929 \\
-5.53602 \\
5.61986 \\
-3.86350 \\
1.79968 \\
-5.48022 \\
9.90367 \\
-8.09626\end{array}$ & $\begin{array}{l}68054 \\
79553 \\
73750 \\
70696 \\
24870 \\
56607 \\
78444 \\
94365 \\
46668 \\
16414\end{array}$ & $\begin{array}{l}88716 \\
19349 \\
95400 \\
83907 \\
48476 \\
15340 \\
35254 \\
91701 \\
06121 \\
79518\end{array}$ & 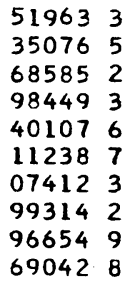 & $\begin{array}{l}(-16) \\
(-13) \\
(-11) \\
(-09) \\
(-07) \\
(-05) \\
(-03) \\
(-02) \\
(-01) \\
(00)\end{array}$ & $\begin{array}{r}-1.41965 \\
1.34852 \\
-8.97874 \\
3.35733 \\
-9.80017 \\
1.76658 \\
-2.58993 \\
1.97161 \\
-1.36202 \\
1.00000\end{array}$ & $\begin{array}{l}98040 \\
03552 \\
17847 \\
07823 \\
32529 \\
33281 \\
67889 \\
45596 \\
98660 \\
00000\end{array}$ & $\begin{array}{l}97653 \\
59192 \\
37323 \\
35633 \\
00364 \\
80539 \\
18623 \\
61601 \\
70096 \\
00000\end{array}$ & $\begin{array}{ll}26071 & 0 \\
68792 & 1 \\
82249 & 4 \\
51346 & 7 \\
84028 & 5 \\
21937 & 9 \\
48909 & 1 \\
18167 & 1 \\
90749 & 2 \\
00000 & 0\end{array}$ & $\begin{array}{l}(-11) \\
(-09) \\
(-08) \\
(-06) \\
(-05) \\
(-03) \\
(-02) \\
(-01) \\
(00) \\
(00)\end{array}$ \\
\hline
\end{tabular}

the computation

$$
\zeta(s)-1=\frac{R_{l m}(s)}{s-1}-1
$$

can lead to considerable subtraction error. Instead, one should use the form

$$
\zeta(s)-1 \simeq \frac{\sum_{i=0}^{M} \hat{p}_{i} s^{i}}{(s-1) \sum_{i=0}^{m} q_{i} s^{i}}, \quad .5 \leqq s \leqq 5,
$$

where

$$
M=\max (l, m+1), \quad \sum_{i=0}^{M} \hat{p}_{i} s^{i} \equiv \sum_{i=0}^{l} p_{i} s^{i}-(s-1) \sum_{i=0}^{m} q_{i} s^{i},
$$

and the $\hat{p}_{i}$ are determined explicitly. It is not difficult to show that if $\delta_{l m}$ is the relative error in using (4.1) as an approximation to $\zeta(s)$, then the relative error in using (4.2) as an approximation to $\zeta(s)-1$ is bounded by $30 \delta_{l m}$. Thus, if the relative error in the machine is bounded by $10^{-D}$, one should choose an $R_{l m}(s), .5 \leqq s \leqq 5$, such that $30 \delta_{l m}<10^{-D}$.

In the second interval, the $R_{l m}(s)$ are poorly conditioned when expressed in the 
usual way. They are therefore presented instead as well-conditioned ratios of sums of Chebyshev polynomials. In the last two intervals, better accuracy in $\zeta(s)-1$ will be obtained by setting $n=[s]$ and computing $2^{-s+(1 / 8) R(1 / 8)}$ as $2^{-n} \times 2^{-(s-n)+(1 / s) R(1 / 8)}$, where $2^{-n}$ can be done exactly on most computers by modifying the floating point exponent. Use of the new self-contained exponentiation routines [1] would also help.

Subroutines for both the CDC 3600 and the IBM 360 have been written using these techniques. In each case, essentially machine precision was achieved for $\zeta(s)$ and $\zeta(s)-1$ for $s$ in the respective ranges.

Argonne National Laboratory

9700 South Cass Avenue

Argonne, Illinois 60439

Department of Computer Science

University of Notre Dame

Notre Dame, Indiana 46556

Applied Mathematics Division

Argonne National Laboratory

Argonne, Illinois 60439

1. N. W. Clark \& W. J. Cody, Self-Contained Exponentiation, Proc. FJCC, AFIPS Press, Montvale, N. J., 1969, pp. 701-706.

2. W. J. CoDy, "A survey of practical rational and polynomial approximation of functions," SIAM Rev., v. 12, 1970, pp. 400-423.

3. R. F. KING \& D. L. PHILlips, "The logarithmic error and Newton's method for the square root," Comm. $A C M$, v. 12, 1969, pp. 87-88.

4. D. H. LeHMER, "On the maxima and minima of Bernoulli polynomials," Amer. Math. Monthly, v. 47, 1940, pp. 533-538. MR 2, 43.

5. B. MARKMAN, "The Riemann zeta function," BIT, v. 5, 1965, pp. 138-141.

6. P. H. Sterbenz \& C. T. FIKE, "Optimal starting approximations for Newton's method," Math. Comp., v. 23, 1969, pp. 313-318. MR 39 \#6511.

7. H. C. ThACHER, JR., "On expansions of the Riemann zeta function," Math. Comp. (To appear.) 